\title{
Understanding Molecular Structures of Buried Interfaces in Halide Perovskite Photovoltaic Devices Nondestructively with Sub-Monolayer Sensitivity Using Sum Frequency Generation Vibrational Spectroscopy
}

\author{
Minүu Xiao, Tieyi Lu, Ting Lin, John S. Andre, and Zhan Chen*
}

As performance of halide perovskite devices progresses, the device structure becomes more complex with more layers. Molecular interfacial structures between different layers play an increasingly important role in determining the overall performance in a halide perovskite device. However, current understanding of such interfacial structures at a molecular level nondestructively is limited, partially due to a lack of appropriate analytical tools to probe buried interfacial molecular structures in situ. Here, sum frequency generation (SFG) vibrational spectroscopy, a state-of-the-art nonlinear interface sensitive spectroscopy, is introduced to the halide perovskite research community and is presented as a powerful tool to understand molecule behavior at buried halide perovskite interfaces in situ. It is found that interfacial molecular orientations revealed by SFG can be directly correlated to halide perovskite device performance. Here how SFG can examine molecular structures (e.g., orientations) at the perovskite/hole transporting layer and perovskite/electron transporting layer interfaces is discussed. This will promote the use of SFC to investigate molecular structures of buried interfaces in various halide perovskite materials and devices in situ nondestructively with a sub-monolayer interface sensitivity. Such research will help to elucidate structure-function relationships of buried interfaces, aiding in the rational design/development of halide perovskite materials/devices with improved performance.

\section{Introduction}

Halide perovskites are among the most promising semiconducting materials for photovoltaic and optoelectronic applications, and have been extensively studied over the past decades. ${ }^{[1]}$ As progress has been made in synthesis of halide perovskite active layers, ${ }^{[2]}$ selection of charge transporting materials, ${ }^{[3]}$ and recipes for device fabrication, ${ }^{[4]}$ little understanding has been established on the buried interfacial molecular structures of perovskite devices. Recently, it has

M. Xiao, T. Lu, T. Lin, J. S. Andre, Z. Chen

Department of Chemistry

University of Michigan

930 North University Avenue, Ann Arbor, MI 48109, USA

E-mail: zhanc@umich.edu

The ORCID identification number(s) for the author(s) of this article can be found under https://doi.org/10.1002/aenm.201903053.

DOI: 10.1002/aenm.201903053 been demonstrated that interfaces play important roles in halide perovskite photovoltaics. ${ }^{[5]}$ Related examples include: 1) interfacial molecular structure of a hole transporting layer (HTL) can greatly impact the overall photoconversion efficiency (PCE) of a halide perovskite photovoltaic device. ${ }^{[6]}$ 2) Interfacial modification of both the electron transporting layer (ETL) and HTL of an unencapsulated halide perovskite photovoltaic device can increase its operational stability to over 1000 h. ${ }^{[7]}$ 3) Interfacial structure can dictate perovskite bulk structurea properly chosen surface underneath the perovskite film can lead to an optimized perovskite bulk structure and promote the perovskite solar cell PCE up to $\approx 19.3 \% .{ }^{[8]}$ In addition to the above examples, it is worth mentioning that recent research also shows that applications of charge defect passivation molecules in interface-near regions, ${ }^{[9]}$ interlayers, ${ }^{[10]}$ and complex 2D/3D interfacial regions ${ }^{[11]}$ can effectively improve the perovskite photovoltaic device performance. Also, recently it was reported that interface recombination is the limiting recombination mechanism in perovskite solar cells, which directly influences the device performance. ${ }^{[12]}$

With the rapid development of halide perovskite materials and devices, it is urgently desired to understand and design different interfaces in perovskite devices for fundamental research and practical applications. However, many interfaces in perovskite devices are buried (e.g., solid/solid interfaces) which are difficult to probe nondestructively. Also, to understand such buried interfaces in depth, it is important to characterize the structures at interfaces at the molecular level, i.e., identify the interfacial functional group composition and determine interfacial functional group orientation.

The critical barrier for buried interfacial molecular structure research is the lack of surface/interfacial sensitive methods to nondestructively examine buried interfaces in situ at the molecular level with monolayer (or sub-monolayer) sensitivity. Monolayer sensitivity is important because the buried interfacial molecular structure is intrinsically the structure of a monolayer at an interface. Unfortunately, most of the techniques 
used to study sample crystallinity are not able to achieve such sensitivity. Nondestructive measurements are critical because halide perovskite active materials are extremely sensitive to contacting air; ${ }^{[13]}$ an ideal in situ interface measurement technique should be applied to probe the buried interface. Some of the commonly adopted interfacial analytical tools for studying halide perovskites include X-ray absorption near edge spectroscopy (XANES), grazing incidence X-ray diffraction (GIXRD), cross-section scanning electron microscopy (SEM), etc. The above analytical techniques using X-rays are based on crystallinity measurements of the materials, which lack monolayer sensitivity, and may not be able to study buried interfaces in situ. XANES and GIXRD can achieve near surface sensitivity $(\approx 7-10 \mathrm{~nm})$, but still lack the ability to reach monolayer sensitivity. Commonly used imaging techniques like SEM can provide morphology of a device's surface, or image a device's crosssection, but do not provide molecular level structural information. To understand the detailed interfacial structures and structure-function relationships, it is necessary to obtain molecular level structural information.

Here, a surface/interface sensitive nonlinear optical spectroscopy, sum frequency generation (SFG) vibrational spectroscopy, which can probe molecular structures of buried interfaces in situ with a sub-monolayer sensitivity, is introduced. SFG is a second-order nonlinear optical process, which involves two incoming beams and an SFG signal beam. For an SFG process to happen, two incoming photons must be spatially and temporally overlapped at the sample to generate a third photon (SFG signal), which combines the overall energies of the two incoming photons. ${ }^{[14-16]}$ SFG has been successfully applied to elucidate molecular structures of buried solid/solid interfaces in situ at the molecular level. ${ }^{[17,18]}$

\section{SFG Introduction}

\subsection{SFG Interface Sensitivity}

SFG vibrational spectroscopy (or SFG in short) is a secondorder nonlinear optical spectroscopy. Early SFG theoretical and experimental foundations have been developed by Shen and co-workers. ${ }^{[14,16]}$ The selection rule of a second-order nonlinear optical process dictates that the SFG signal can only be generated from a medium without inversion symmetry (see more detailed discussion in Section 2.3). Most bulk materials possess inversion symmetry (or are central symmetric-symmetric with respect to an inversion center), therefore no SFG signal can be generated. Due to the broken inversion symmetry at surfaces or interfaces, SFG can be used to selectively study molecules on surfaces and at buried interfaces. Extensive research indicates that SFG is "extremely" surface/interface sensitive, down to a sub-monolayer. ${ }^{[15]}$

\subsection{Capability of SFG to Probe Buried Interfaces In Situ Nondestructively}

Figure 1a shows a schematic of SFG signal generation from a sample surface. In a typical SFG experiment, a visible (e.g., 532 or

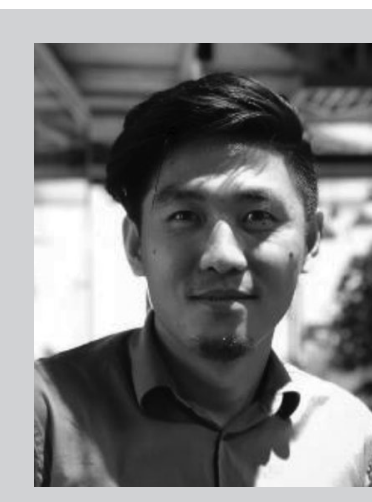

Minyu Xiao received his BS degree in Chemistry from the Nanjing University and Ph.D. degree in the Department of Chemistry, University of Michigan. He has studied molecular structures of semiconducting polymers and molecular interactions on 2D materials as a graduate student. Currently he is working at McKinsey \& Company in Shanghai.

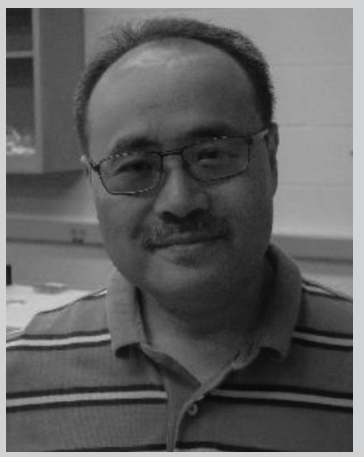

Zhan Chen received his Ph.D. degree in chemistry from the University of California at Berkeley and did his postdoctoral research at the Lawrence Berkeley National Laboratory. Currently he is a professor of chemistry, macromolecular science and engineering, applied physics, and biophysics at the University of Michigan. He is interested in molecular level studies on complex surfaces and interfaces using nonlinear optical spectroscopic techniques.

$800 \mathrm{~nm}$ ) beam and an infrared (IR) beam (frequency tunable or broadband) are used as input beams. As long as these two beams can reach a buried interface, SFG signal can be generated from said buried interface. As we discussed, the bulk media above and below this buried interface usually (because of possessing inversion symmetry) would not generate SFG signal due to the SFG selection rule. Therefore SFG can selectively probe a buried solid/ solid interface in situ nondestructively using a reflection sample geometry, without the need to break the interface, ${ }^{[17]}$ including the buried interfaces involving perovskite materials. ${ }^{[6]}$

\subsection{Capability of SFG to Provide Molecular Level Structural Information}

\subsubsection{Identification of Interfacial Functional Groups}

Figure 1b illustrates the energy level diagram of an SFG process, indicating that SFG is a combined process of infrared absorption and anti-Stoke Raman scattering. In our SFG experiments, the wavenumber of the input IR beam is continuously tuned and the SFG signal is detected as a function of the input IR beam wavenumber. When the IR beam is tuned to a resonance frequency of a functional group's vibrational mode at the interface, the SFG signal intensity can be enhanced. Therefore the SFG signal intensity plotted versus the input IR beam wavenumber provides the vibrational spectrum of the interface. A vibrational spectrum 

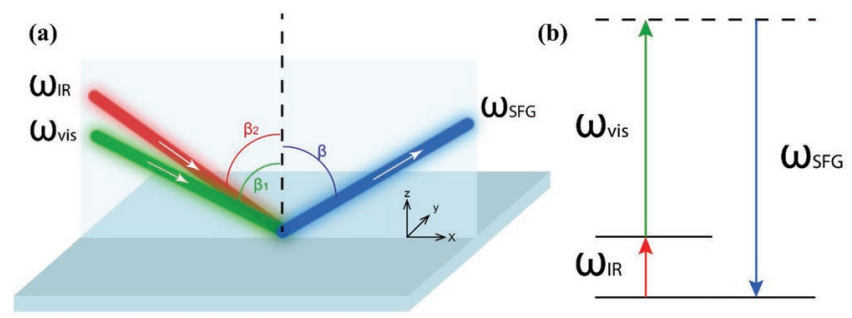

Figure 1. a) SFG signal generation, and b) SFG energy level diagram.

can be thought to show the finger prints of a molecule. The peak centers in the spectrum can be used to identify various interfacial functional groups, because different functional groups have characteristic vibrational modes with different resonance wavenumbers, leading to peaks with different center positions. Therefore SFG can be used to identify interfacial species or compositions.

\subsubsection{Orientation Determination of Interfacial Functional Groups}

Figure 1b shows the energy level diagram for an SFG process. An SFG process can be considered as a combined IR absorption (red arrow in Figure 1b) and anti-Stokes Raman scattering (green and blue arrows in Figure 1b). The signal intensity of IR absorption and Raman scattering of a vibrational mode are determined by the infrared transition dipole moment and Raman polarizability tensor of the vibrational mode respectively. The SFG signal intensity of the vibrational mode is determined by the hyperpolarizability, which is a product of the infrared transition dipole moment and Raman polarizability tensor (Equation (1)). ${ }^{[17]}$ In Equation (1), $\frac{\partial \mu_{c}}{\partial Q_{q}}$ and $\frac{\partial \alpha_{a b}}{\partial Q_{q}}$ are the infrared transition dipole moment and Raman polarizability tensor, respectively

$\beta_{a b c}^{(2)}=-\frac{1}{2 \varepsilon_{0} \omega_{q}} \frac{\partial \alpha_{a b}^{*}}{\partial Q_{q}} \frac{\partial \mu_{c}}{\partial Q_{q}}$

An SFG experiment probes the overall effects of the hyperpolarizability of all the molecules at the interface, or the second order nonlinear optical susceptibility $\chi^{(2)}$, which is a third rank tensor. Before we discuss the orientation determination from $\chi^{(2)}$ measurements, we want to revisit the SFG interface sensitivity. $\chi^{(2)}$ is a polar tensor, which means that it will change sign under an inversion operation. For a medium with inversion symmetry, such as a bulk material, any property including $\chi^{(2)}$ is the same under an inversion operation. Therefore for a medium with inversion symmetry, $\chi^{(2)}$ can only be zero, and therefore no SFG signal can be generated. Most bulk materials of organic molecules and polymers usually possess inversion symmetry, therefore no SFG signal can be detected from such bulk materials. At a surface/interface where inversion symmetry is broken, $\chi^{(2)}$ can be nonzero and SFG signal can be collected.

It is important to measure the orientations of different functional groups at interfaces in halide perovskite photovoltaic devices. As we will present below, the orientation of interfacial functional groups at the buried perovskite/semiconducting polymer HTL interfaces is strongly linked to the perovskite solar cell PCE. ${ }^{[6]}$ Optimization of such interfacial orientations can greatly enhance the perovskite solar cell PCE to record high values. ${ }^{[19]}$ Here we will present some details regarding the SFG orientation analysis due to the significant effects interfacial molecular orientation has on halide perovskite device performance. SFG can examine many interfacial chemical functional groups including $\mathrm{O}-\mathrm{H}$ (hydroxyl), $-\mathrm{CH}_{3}$ (methyl), $-\mathrm{CH}_{2}$ (methylene), aromatic $\mathrm{C}-\mathrm{H}, \mathrm{C}=\mathrm{O}$ (carbonyl), $\mathrm{C}=\mathrm{C}$ (e.g., in thiophenes, which will be discussed in detail below), amide I, C-F groups, etc. through their vibrational modes. SFG spectra collected using different polarization combinations probe different SFG susceptibility tensor elements, from which the orientation of a functional group or molecule can be determined. Basically, collecting optical spectra using varied polarizations measures the projections of the studied molecules (vibrational modes) onto different axes in the lab-fixed coordination frame. From these projections, molecular or functional group orientation can be determined. Detailed theoretical fundamentals for SFG orientation analysis have been studied extensively by Hirose et al., ${ }^{[20,21]}$ Shen and co-workers, ${ }^{[16]}$ and Moad and Simpson. ${ }^{[22]}$

In more detail, using different polarization combinations of the input and output beams in the SFG experiment, different elements of the $\chi^{(2)}$ tensor can be accessed (measured). For example, using ssp (s-polarized SFG signal, s-polarized visible input beam, and p-polarized IR beam) polarization combination, $\chi_{\text {eff, ssp }}^{(2)}$ can be measured. Such measured elements can be correlated to the elements of the $\chi^{(2)}$ defined in the lab coordination $(x y z)$ system through the following equations

$$
\begin{aligned}
& \chi_{\mathrm{eff}, \text { ssp }}^{(2)}=\left[L_{Y Y}(\omega) L_{\gamma Y}\left(\omega_{1}\right) L_{z z}\left(\omega_{2}\right) \sin \beta_{2}\right] \chi_{Y Y z}^{(2)} \\
& \chi_{\mathrm{eff}, \mathrm{sps}}^{(2)}=\left[L_{Y Y}(\omega) L_{z z}\left(\omega_{1}\right) L_{Y Y}\left(\omega_{2}\right) \sin \beta_{1}\right] \chi_{Y z Y}^{(2)} \\
& \chi_{\mathrm{eff}, \mathrm{pss}}^{(2)}=\left[L_{z z}(\omega) L_{Y Y}\left(\omega_{1}\right) L_{Y Y}\left(\omega_{2}\right) \sin \beta\right] \chi_{z Y Y}^{(2)} \\
& \chi_{\text {eff }, \mathrm{ppp}}^{(2)}=-\left[L_{x x}(\omega) L_{x x}\left(\omega_{1}\right) L_{z z}\left(\omega_{2}\right) \cos \beta \cos \beta_{1} \sin \beta_{2}\right] \chi_{x x z}^{(2)} \\
& -\left[L_{x x}(\omega) L_{z z}\left(\omega_{1}\right) L_{x x}\left(\omega_{2}\right) \cos \beta \sin \beta_{1} \cos \beta_{2}\right] \chi_{x z x}^{(2)} \\
& +\left[L_{z z}(\omega) L_{x x}\left(\omega_{1}\right) L_{x x}\left(\omega_{2}\right) \sin \beta \cos \beta_{1} \cos \beta_{2}\right] \chi_{z x x}^{(2)} \\
& +\left[L_{z z}(\omega) L_{z z}\left(\omega_{1}\right) L_{z z}\left(\omega_{2}\right) \sin \beta \sin \beta_{1} \sin \beta_{2}\right] \chi_{z z z}^{(2)}
\end{aligned}
$$

The $L$ terms in the above equations are Fresnel coefficients, which relate the input/output beam fields to the SFG signal intensity. At an azimuthally isotropic interface, only four independent $\chi^{(2)}$ elements $\left(\chi_{i j k}\right)$ are nonzero

$\chi_{x x z}^{(2)}=\chi_{y Y z}^{(2)}, \chi_{x z x}^{(2)}=\chi_{y z y}^{(2)}, \chi_{z x x}^{(2)}=\chi_{z y y}^{(2)}, \chi_{z z z}^{(2)}$

Therefore, only selected polarizations measuring the corresponding tensor components lead to nonzero signals in SFG experiments (as shown in Equation (6)). ${ }^{[17]}$

The SFG measured $\chi^{(2)}$ components in the lab-fixed coordination system are the overall effects of related hyperpolarizability elements of all the molecules. For a particular vibrational mode of a functional group, $\chi^{(2)}$ is correlated to the hyperpolarizability of all the molecules at the interface through molecular orientation, since hyperpolarizability is defined in the molecule-fixed coordination system while $\chi^{(2)}$ is measured in the lab-fixed coordination system. Such correlations also depend on the symmetry of the vibrational mode under study. Here we will discuss the two commonly encountered functional groups 
in halide perovskite systems as examples for SFG orientation analysis: 1) methyl group $\left(-\mathrm{CH}_{3}\right)$ and 2$) \mathrm{C}=\mathrm{C}$ bonds in thiophene ring. Figure 2 shows an illustration of the tilt angle and twist angle of a methyl group and a thiophene ring in a labfixed frame, respectively.

The methyl group is the most widely studied functional group with SFG, and exists in halide perovskite systems, as well as in solution processed organic semiconducting molecules (e.g., poly-3-hexylthiophene or P3HT, an HTL material[ ${ }^{[23]}$ ) used in perovskite solar cells. A methyl group is usually treated with a $\mathrm{C}_{3 \mathrm{~V}}$ symmetry (Figure $2 \mathrm{a}$ ) when doing orientation analysis.

For the symmetric $\mathrm{C}-\mathrm{H}$ stretch of a methyl group with a $\mathrm{C}_{3 \mathrm{v}}$ symmetry ${ }^{[20]}$

$$
\begin{aligned}
& \chi_{x x z, s}^{(2)}=\chi_{y Y z, s}^{(2)}=\frac{1}{2} N \beta_{c c c}^{(2)}\left[(1+r)\langle\cos \theta\rangle-(1-r)\left\langle\cos ^{3} \theta\right\rangle\right] \\
& \chi_{x z x, s}^{(2)}=\chi_{y z y, s}^{(2)}=\chi_{z x x, s}^{(2)}=\chi_{z y y, s}^{(2)}=\frac{1}{2} N \beta_{c c c}^{(2)}(1-r)\left[\langle\cos \theta\rangle-\left\langle\cos ^{3} \theta\right\rangle\right] \\
& \chi_{z z z, s}^{(2)}=N \beta_{c c c}^{(2)}\left[r\langle\cos \theta\rangle+(1-r)\left\langle\cos ^{3} \theta\right\rangle\right]
\end{aligned}
$$

For the asymmetric $\mathrm{C}-\mathrm{H}$ stretch of $\mathrm{C}_{3 \mathrm{v}}$ symmetry

$$
\begin{aligned}
& \chi_{y y z, a s}^{(2)}=\chi_{x x z, a s}^{(2)}=-\frac{1}{2} N \beta_{c a a}^{(2)}\left[\langle\cos \theta\rangle-\left\langle\cos ^{3} \theta\right\rangle\right] \\
& \chi_{z x x, a s}^{(2)}=\chi_{z y y, a s}^{(2)}=\chi_{y z y, a s}^{(2)}=\chi_{x z x, a s}^{(2)}=\frac{1}{2} N \beta_{a c a}^{(2)}\left\langle\cos ^{3} \theta\right\rangle \\
& \chi_{z z z, a s}^{(2)}=N \beta_{c a a}^{(2)}\left[\langle\cos \theta\rangle-\left\langle\cos ^{3} \theta\right\rangle\right]
\end{aligned}
$$

Here $\theta$ is the tilt angle, the angle between the surface normal and the main axis of the functional group (here the methyl

(a)

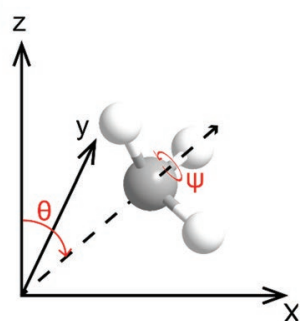

(c) $\left|\chi_{y y z, a s}\right| \chi_{z z z}$

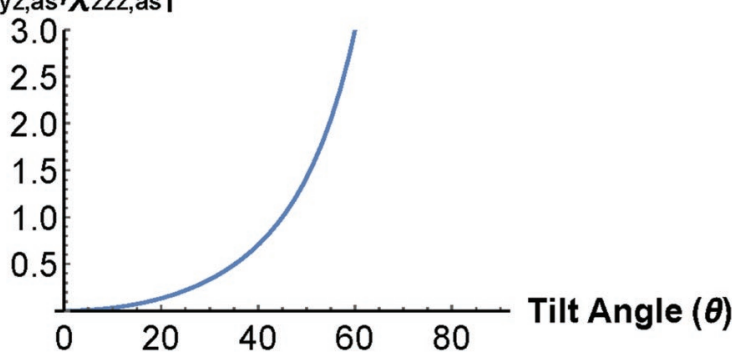

Figure 2. Tilt angle $\theta$ and twist angle $\psi$ of a) a methyl group and b) a thiophene ring in the lab-fixed frame. c) $\left|\chi_{y y z, a s}\right| \chi_{z z z, a s} \mid$ plot of $C_{3 v}$ asymmetric stretch (e.g., for a methyl group) with a delta distribution. group). “ \langle\rangle " means average. The twist angle $\psi$ can be averaged out for $\mathrm{C}_{3 \mathrm{v}}$ symmetry. In the above equations, as we discussed, different elements in the $\chi^{(2)}$ tensor can be measured using SFG signals collected using different polarizations. Different elements in the hyperpolarizability $\left(\beta_{a b c}^{(2)}\right.$ terms) can be deduced by calculating (or measuring) IR transition dipole and Raman polarizability. If the number of the molecules probed can be quantified ( $\mathrm{N}$ in the above equations), then the orientation angle information can be obtained. Usually it is difficult to determine the value of $\mathrm{N}$, therefore the ratios of the different $\chi^{(2)}$ elements ( $\mathrm{N}$ is canceled) are used for orientation analysis. Figure 2c displays the correlation between the measured $\left|\chi_{y y z, a s}\right| \chi_{z z z, a s} \mid$ ratio of the $\mathrm{C}_{3 \mathrm{v}}$ asymmetric stretch and the orientation angle of the methyl group. Typically a delta or Gaussian orientation distribution is assumed. From this correlation and the experimentally measured $\left|\chi_{y y z, a s}\right| \chi_{z z z, a s} \mid$ ratio, the methyl orientation can be deduced. Using similar strategies, correlations between various $\chi^{(2)}$ component ratios and orientation angles of different functional groups can be deduced, which can be used to determine the orientation of such functional groups according to the experimentally measured ratios. Such orientation analysis will be used to study thiophene ring orientation which will be discussed in detail below.

The thiophene ring, one of the most widely available functional groups in organic semiconductors, adopts a $\mathrm{C}_{2 \mathrm{v}}$ symmetry (Figure 2b). ${ }^{[6]}$ For the thiophene ring, both tilt angle and twist angle are needed to describe the five-membered ring orientation. If we also assume that the twist angle is random and can be averaged, then for the symmetric stretch of $\mathrm{C}_{2 \mathrm{v}}$ symmetry

$$
\begin{aligned}
\chi_{x x z, s}^{(2)}= & \chi_{y y z, s}^{(2)}=\frac{1}{4} N\left(\beta_{a a c}^{(2)}+\beta_{b b c}^{(2)}+\beta_{c c c}^{(2)}\right)\langle\cos \theta\rangle \\
& +\frac{1}{4} N\left(\beta_{a a c}^{(2)}+\beta_{b b c}^{(2)}+\beta_{c c c}^{(2)}\right)\left\langle\cos ^{3} \theta\right\rangle \\
\chi_{x z x, s}^{(2)}= & \chi_{z x x, s}^{(2)}=\chi_{y z y, s}^{(2)}=\chi_{z \gamma y, s}^{(2)} \\
= & -\frac{1}{4} N\left(\beta_{a a c}^{(2)}+\beta_{b b c}^{(2)}-2 \beta_{c c c}^{(2)}\right)\left(\langle\cos \theta\rangle-\left\langle\cos ^{3} \theta\right\rangle\right) \\
\chi_{z z z, s}^{(2)}= & \frac{1}{2} N\left(\beta_{a a c}^{(2)}+\beta_{b b c}^{(2)}\right)\langle\cos \theta\rangle \\
& -\frac{1}{2} N\left(\beta_{a a c}^{(2)}+\beta_{b b c}^{(2)}-2 \beta_{c c c}^{(2)}\right)\left\langle\cos ^{3} \theta\right\rangle
\end{aligned}
$$

For the asymmetric stretch of $\mathrm{C}_{2 \mathrm{v}}$ symmetry

$$
\begin{aligned}
& \chi_{x x z, a s}^{(2)}=\chi_{y y z, a s}^{(2)}=-\frac{1}{2} N \beta_{a c a}^{(2)}\left[\langle\cos \theta\rangle-\left\langle\cos ^{3} \theta\right\rangle\right] \\
& \chi_{x z x, a s}^{(2)}=\chi_{z x x, a s}^{(2)}=\chi_{y z y, a s}^{(2)}=\chi_{z \gamma y, a s}^{(2)}=\frac{1}{2} N \beta_{a c a}^{(2)}\left\langle\cos ^{3} \theta\right\rangle \\
& \chi_{z z z, a s}^{(2)}=N \beta_{a c a}^{(2)}\left[\langle\cos \theta\rangle-\left\langle\cos ^{3} \theta\right\rangle\right]
\end{aligned}
$$

In summary, to probe the molecular structure of a buried interface, SFG spectral peak positions are used to determine the interfacial composition (presence of which functional group at the interface). From SFG spectra collected with different 
polarizations, we can determine the ratios of different $\chi^{(2)}$ tensor elements. According to the symmetry of a functional group, we can know the correlation between the measured $\chi^{(2)}$ tensor element ratios and the allowed functional group orientations. The functional group interfacial orientation can then be deduced from such correlations and the measured ratios. Therefore SFG can probe the molecular structure of a buried interface in situ nondestructively with a sub-monolayer sensitivity.

\section{SFG Studies on Interfacial Structures of Halide Perovskite Devices}

Extensive halide perovskite characterization research has focused on the structure of halide perovskite, especially bulk crystallinities. As more understandings of halide perovskite materials are developed, the optimization of the structures of hole transporting materials (HTMs) and electron transporting materials (ETMs) becomes more important to further improve device performance. For organic HTMs and ETMs (e.g., P3HT, Spiro-OMeTad, etc.), improving their bulk crystallinities is intrinsically difficult, and one should consider optimizing the interfacial structures between HTMs or ETMs and the halide perovskite layer based on in-depth understanding on such interfacial structures and related structure-property relationships.

As discussed, SFG is a powerful technique that probes buried interfacial structures at the molecular level through examining vibrational modes of various functional groups (especially for organic materials). Many halide perovskite containing devices have incorporated various $\mathrm{n}$-type and $\mathrm{p}$-type organic semiconducting materials (e.g., P3HT, Spiro-OMETAD, PCBM, PTB7, etc.) which can be investigated using SFG. Recent advances in organic semiconductors related to perovskite photovoltaic devices using SFG will be discussed in this section. We hope that this discussion serves as a general introduction of the SFG applications in halide perovskite related research.

\subsection{Effect of Molecular Orientation at the HTL/Halide Perovskite Interface on Perovskite Photovoltaic Device PCE}

To the best of our knowledge, we published the first study that reported the interfacial molecular orientation in a perovskite photovoltaic device. ${ }^{[6]}$ We found a standing-up polythiophene (PT) backbone at the interface between PT (serving as HTM) and halide perovskite benefits the current density and hole transportation. Our results indicate that a device with a standing-up HTM molecular orientation at the HTM/perovskite interface showed a short-circuit current density $\left(U_{\text {sc }}\right)$ of $\approx 19.97 \mathrm{~mA} \mathrm{mc}^{-2}$, while a similar device with lying-down interfacial HTM molecules at the HTM/perovskite interface showed a much lower $J_{\mathrm{sc}}$ of $\approx 9.3 \mathrm{~mA} \mathrm{mc^{-2 }}$. Therefore, the overall device power conversion efficiency can be increased by over $100 \%$ when the interfacial molecular orientation of the HTM changed from a lying-down to a standing-up orientation. ${ }^{[6]}$

In more detail, the above halide perovskite photovoltaic devices were fabricated as following: $\mathrm{TiO}_{x}$ serves as ETM, a halide perovskite serves as the photoactive layer, and PT derivatives serve as HTMs. PTs were carefully chosen to only have altered alkyl-side chain lengths. Four PT materials were studied: a) P3HT (with a 6-carbon side chain), P3OT (with an 8-carbon side chain), P3DT (with a 10-carbon side chain), and P3DDT (with a 12-carbon side chain). We showed that the bandgaps of these different PTs are the same, independent of the side chain length. We further confirmed that these PTs have similar bulk structure/properties by measuring the bulk crystallinity with GIXRD and bulk charge carrier conductivity by space-charge limited currents. However, the PCEs of the perovskite photovoltaic devices prepared with four PTs are markedly varied, which we believe was caused by varied interfacial structures between the perovskite layer and the PT HTL. SFG was then applied to examine buried interfacial structure between different PTs and halide perovskite in situ nondestructively.

As discussed above, SFG can be used to probe a buried interfacial structure which can be accessed by the input laser beams in the SFG experiment. It is more challenging to probe the buried perovskite/PT interface in a real photovoltaic device. To model such an interface, we prepared the perovskite/PT interface in a three-layered sample (perovskite/PT/poly(methyl methacrylate) (PMMA)) with the same procedure as that of the real device. $\mathrm{SFG}$ spectra from $\mathrm{C}=\mathrm{C}$ stretching in the thiophene ring $\left(\mathrm{C}_{2 \mathrm{v}}\right.$ symmetry) were collected from the three-layered samples using different polarization combinations (Figure 3). Spectra collected contain signal contributions from PT at the perovskite/PT and PT/PMMA interfaces, from which the SFG signals from the perovskite/PT interface can be deconvoluted by measuring SFG spectra of PT films with different thicknesses. ${ }^{[6]}$

The method to deconvolute SFG signals contributed from a particular interface from SFG signals collected from a sample containing two interfaces has been well developed and will be presented here briefly. Figure 4 shows the SFG signal collected from buried interfaces A and B. ${ }^{[18]}$ Using the ssp SFG signal as an example, we have

$$
\begin{gathered}
\chi_{\text {eff }, \text { ssp }}^{(2)}=L_{\gamma Y}^{\text {interface A }}(\omega) L_{\gamma Y}^{\text {interface A }}\left(\omega_{1}\right) L_{z z}^{\text {interface A }}\left(\omega_{2}\right) \sin \beta_{2} \chi_{y \gamma z}^{\text {interface A }} \\
+L_{\gamma Y}^{\text {interface B }}(\omega) L_{\gamma Y}^{\text {interface B }}\left(\omega_{1}\right) L_{z z}^{\text {interface B }}\left(\omega_{2}\right) \sin \beta_{2} \chi_{y Y z}^{\text {interface B }} \\
+\chi_{\mathrm{NR}} e^{i \varphi_{\text {ssp }}}
\end{gathered}
$$

Based on the thickness of the sample layer 2 (PT in our study) and the refractive indices of various layers, the overall Fresnel coefficients $\left(L_{i i}(i=x, y\right.$, or $z)$ terms) are deduced. According to the measured overall SFG signal, $\chi_{y y z}^{\text {interface } B}$ can be determined. Using different $\chi^{(2)}$ elements obtained from SFG signals collected using different polarization combinations, the PT thiophene orientation at the buried interface between PT and perovskite can be determined.

Figures 5a shows an example of the deconvoluted ssp SFG spectra contributed from the buried interfaces of a $100 \mathrm{~nm}$ P3DDT film. Other SFG spectra shown in Figure 3 can also be analyzed using the same method to obtain signal contributions from each buried interface of various PT derivatives. Figure 5b shows the measured SFG $\chi_{\text {yyz }} / \chi_{\text {yzy }}$ ratios for the PT ring $\mathrm{C}=\mathrm{C}$ stretching mode at the PT/perovskite interface deduced from the deconvoluted ssp and sps SFG spectra. Since the dipole moment of the $\mathrm{C}=\mathrm{C}$ stretching mode is more or less perpendicular to the thiophene ring, it is clear, from Figure $5 \mathrm{~b}$, that a PT with shorter alkyl-side chain stands up at the interface, 
(a)
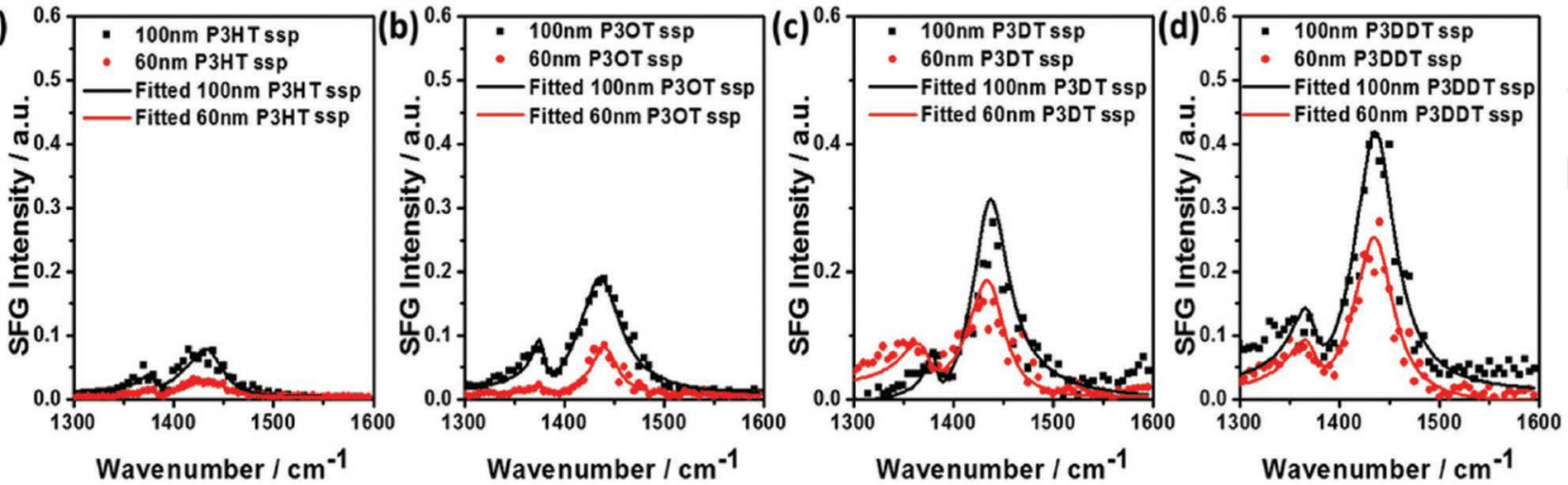

(e)
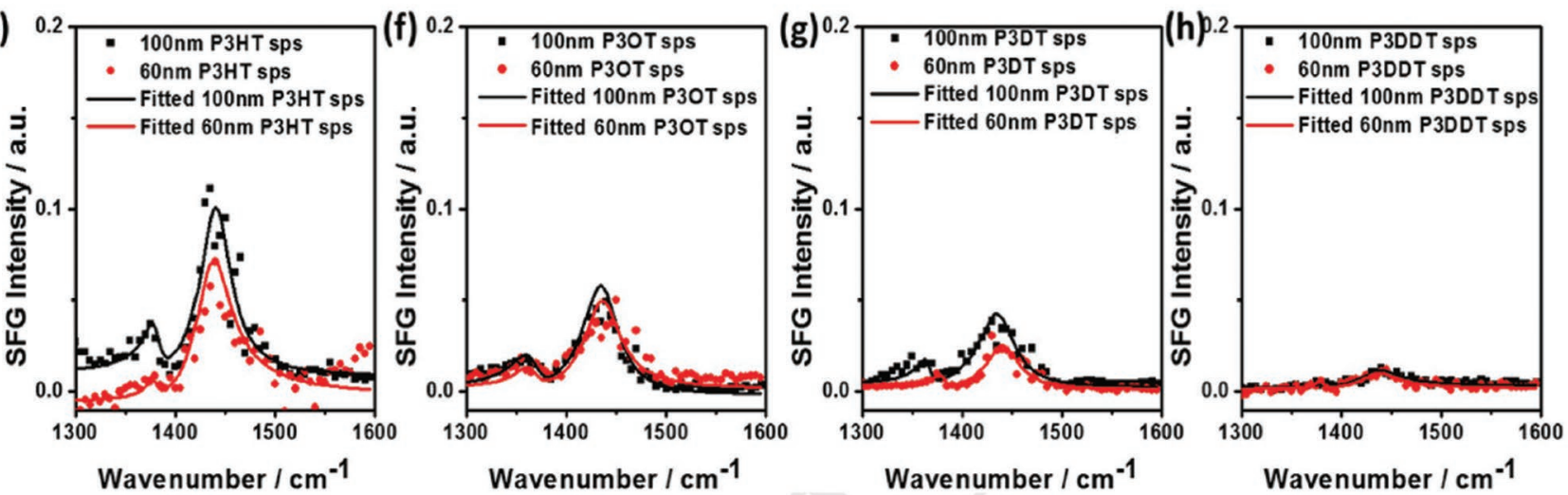

Figure 3. SFG ssp spectra collected from a PT thin film sandwiched between PMMA and perovskite: a) P3HT, b) P3OT, c) P3DT, and d) P3DDT. SFG sps spectra collected from the same samples: e) P3HT, f) P3OT, g) P3DT, and h) P3DDT. Reproduced with permission. ${ }^{[6]}$ Copyright 2017, American Chemical Society.

and a PT with a long alkyl-side chain lies down at the interface. The tilt angles of PTs with intermediate side chain lengths are between the standing-up and lying-down cases. We fabricated perovskite photovoltaic devices using the PTs of different side chain lengths as HTMs and measured their PCEs. Correlating the interfacial PT orientation to the photovoltaic device performance, we found that a standing-up PT backbone orientation at the PT/perovskite interface is a benefit to the overall interfacial hole extraction via improved hole/electron separation at the interface (Figure 6a), leading to a high conversion efficiency

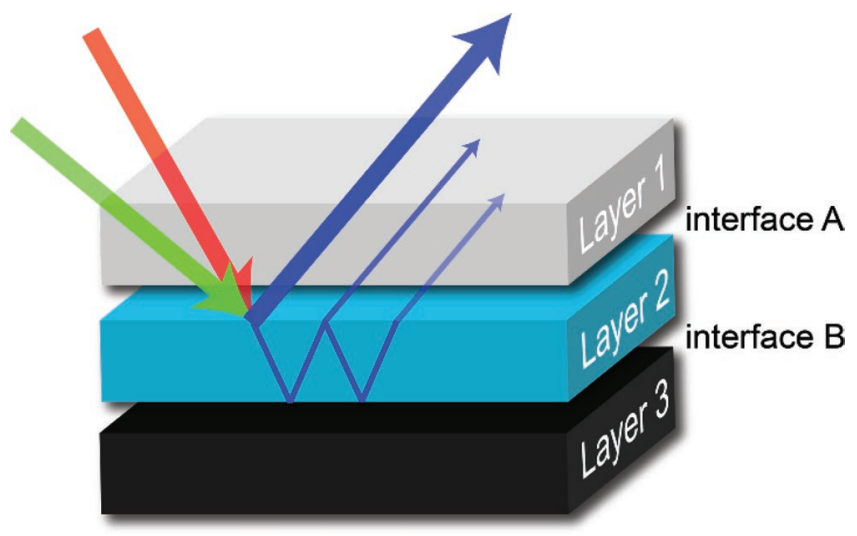

Figure 4. SFG signal from a buried interface in a multilayered sample.
(P3HT: 9.64\%). By contrast, a lying-down interfacial PT chain at the buried interface is likely to cause hole/electron recombination due to overlapping of HOMO/LUMO orbitals of the halide perovskite layer and HTMs, leading to a low conversion efficiency (P3DDT: 3.91\%) (Figure 6b). The orientations of the PTs with intermediate side chain lengths (P3OT and P3DT) were measured to be between P3HT and P3DDT; their perovskite photovoltaic devices' PCEs were also in between, at $6.86 \%$ and $6.64 \%$, respectively. ${ }^{[6]}$

Building on our discovery that a standing-up PT orientation at the HTM/perovskite interface benefits interfacial hole extraction, Zhang et al. successfully designed a novel polythiophenebased HTM copolymer, dithiophene-benzene (DTB, Figure 7a, inset), and their group believes that the DTB molecular backbone can adopt a standing-up pose at the HTM/perovskite interface. ${ }^{[19]}$ The performance of the perovskite photovoltaic device using this designed PT based HTM was excellent, as shown in Figure 7. Its PCE was measured to be $19.68 \%$, which is the highest value for mesoporous perovskite solar cell devices based on dopant-free polymer HTMs reported. ${ }^{[19]}$ Such an inspiring follow-up finding further emphasizes the importance of interfacial molecular orientation for a halide perovskite device's overall performance. It also validated the significance of SFG results obtained from interfacial studies involving perovskite, showing that the use of SFG can guide researchers working with perovskite materials and devices to improve performance. 

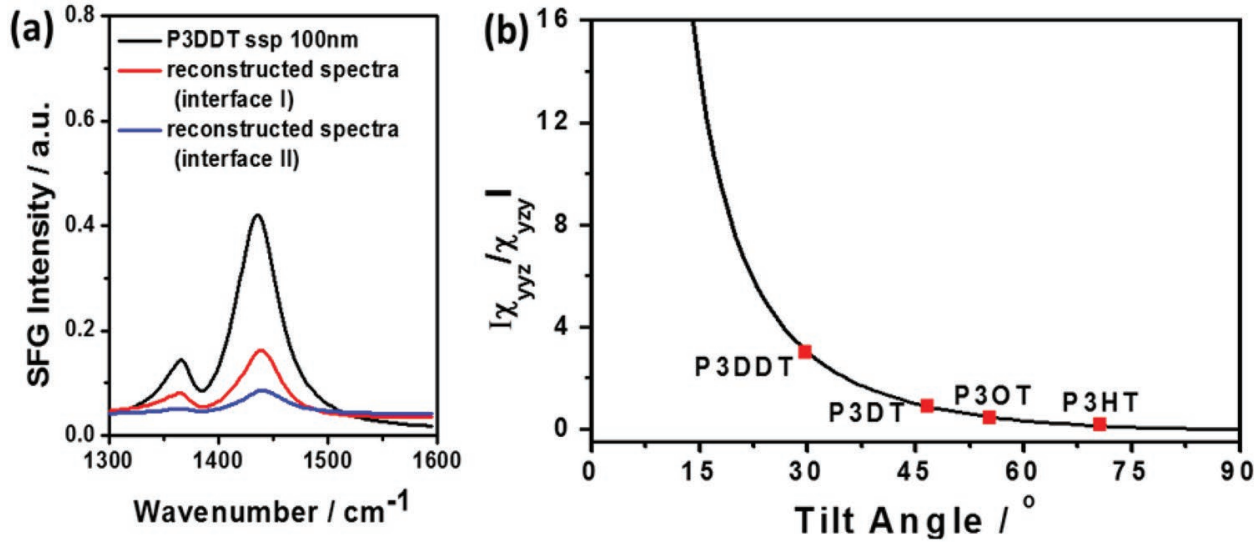

Figure 5. a) Reconstructed ssp spectra, including the overall detected spectrum and the deconvoluted spectra for interfaces I and II for P3DDT film of $100 \mathrm{~nm}$. b) $\chi_{\text {yyz }} / \chi_{y z y}$ ratio as a function of the tilt angle of the net transition dipole of the thiophene ring $\mathrm{C}=\mathrm{C}$ stretch (with respect to the surface normal). Adapted with permission. ${ }^{[6]}$ Copyright 2017, American Chemical Society.

\subsection{Effect of Interfacial Orientation of Small Molecules at the HTL/Halide Perovskite Interface on Solar Cell Power Conversion Efficiency}

Another exciting work using SFG to study perovskite photovoltaic devices was published in late 2018 by Magomedov et al. ${ }^{[24]}$ In this research, SFG was used to characterize self-assembled monolayers (SAMs) with mixed compositions at the buried HTM/perovskite interface. It was found that a mixed SAM at the $\mathrm{HTM}$ /perovskite interface is more disordered, while photovoltaic devices fabricated with disordered SAMs have higher open-circuit voltages $\left(V_{\text {oc }}\right)$, and ultimately higher overall PCEs. ${ }^{[24]}$

Polymeric materials (P3HT, PTAAs, PEDOT:PSS, etc.) and inorganic metal oxides have been commonly used as HTMs. Magomedov et al. developed novel mixed composition SAM molecules (V1036, C4) to functionalize ITO surfaces. ${ }^{[24]}$ They carefully compared the pure V1036 surface, mixed V1036:C4 surface, and pure C4 surface to analyze the SAM surface for structural differences. Solar cells with different SAM compositions were constructed and the device performance was measured. Molecular formulas of V1036 and C4 molecules are shown in Figure $8 \mathrm{c}$,d and can be found in the original paper. Examining the molecular formula for V1036, it is clear that the $\mathrm{C}-\mathrm{N}$ stretch and the $\mathrm{C}=\mathrm{C}$ symmetric stretch in its aromatic ring system, and the $-\mathrm{OCH}_{3}$ (methoxy) groups on its alkyl side

(a)

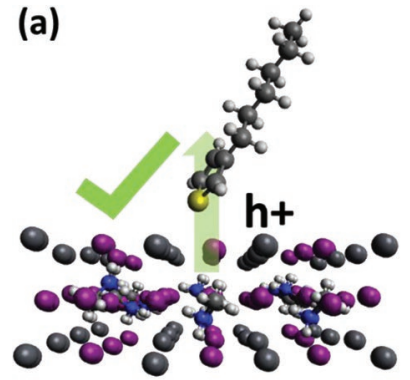

(b)

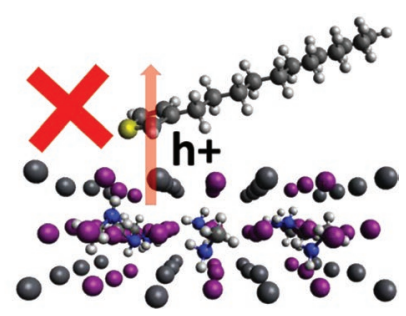

Figure 6. Illustration of hole extraction ability of a) PTs with shorter alkyl side chain and b) PTs with longer alkyl side chain at the HTL/perovskite interface. Reproduced with permission. ${ }^{[6]}$ Copyright 2017, American Chemical Society. chain should be detectable with SFG. From the C4 molecular formula, $-\mathrm{CH}_{2}$ (methylene) groups and $-\mathrm{CH}_{3}$ (methyl) groups can be detected with SFG. Using FTIR absorbance, the authors assigned the peaks for $\mathrm{C}-\mathrm{N}$ stretch at $\approx 1200 \mathrm{~cm}^{-1}, \mathrm{C}=\mathrm{C}$ stretch at $\approx 1500 \mathrm{~cm}^{-1}$. They did not focus on the studies of $-\mathrm{CH}_{3}$ and $-\mathrm{CH}_{2}$ symmetric/asymmetric stretching modes between $\approx 2800$ and $3000 \mathrm{~cm}^{-1}$, possibly due to the potential peak overlaps between V1036 and C4. Figure 8a,b shows that distinct SFG signals can be observed from the pure V1036 SAM surface, including signals from both the $\mathrm{C}-\mathrm{N}$ stretch at $\approx 1237 \mathrm{~cm}^{-1}$ and the $\mathrm{C}=\mathrm{C}$ stretch at $\approx 1488 \mathrm{~cm}^{-1}$. However, such signals were not observed on the mixed V1036:C4 surface. As indicated above, SFG signal is influenced by the number of interfacial molecules probed (value $\mathrm{N}$ in Equations (7)-(18)) and their molecular orientation. The surface coverages of V1036 on the SAM surfaces were measured via IR absorbance intensity to confirm SAM molecules were at the interface. Therefore the absence of the SFG signals is because either the mixed SAM molecules at the interface are disordered or lie down. The conclusion was that a mixed composition SAM surface could lead to a more disordered structure (random orientation) compared to a single composition SAM surface. Photovoltaic devices were constructed with perovskite and SAMs with various compositions ranging from 5\% V1036+95\% C4 to 100\% V1036 and the $J-V$ characteristics were measured. Results from these measurements showed that devices with the most mixed SAM surfaces (excluding the 5\% V1036 case) have higher overall PCEs compared to that prepared with the pure V1036. This indicates that a more disordered small molecule orientation at the HTM/ perovskite interface is preferred for better overall PCEs for perovskite solar cells. ${ }^{[24]}$ We think that finding by Magomedov et al. on the effect of disordered small molecules at the HTM/ perovskite interface is exciting.

3.3. SFG Studies on Other Important Semiconducting Materials Used in Perovskite Photovoltaic Devices

Besides SFG studies on interfaces in perovskite photovoltaic devices, SFG research is actively investigating interfacial 

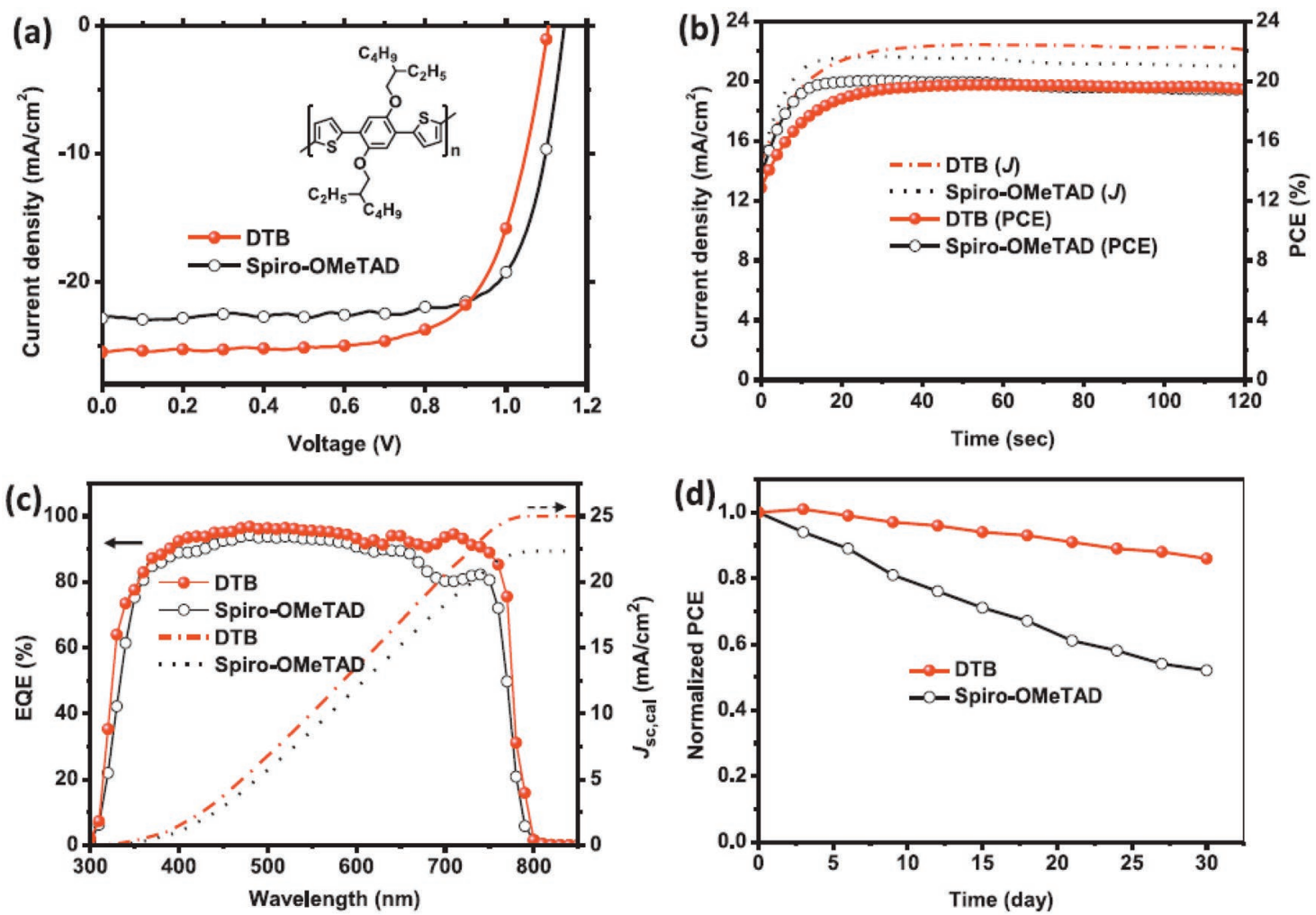

Figure 7. a) $J-V$ characteristics with DTB molecular formula in the inset, b) stabilized power outputs at the maximum power point, c) external quantum efficiency (EQE) charts, and d) evolution of PECs in air for the solar cell devices. Adapted with permission. ${ }^{[19]}$ Copyright 2018, Wiley.

molecular behavior of semiconducting materials, which are commonly used in perovskite photovoltaic devices. For example, Xiao et al. showed that different solvents used to prepare thin films of solvent processable organic semiconducting materials led to different molecular structures of the film at both the surface and buried interface. ${ }^{[25]}$ Variation of the solvent composition is a common approach used to prepare thin films in perovskite photovoltaic devices to optimize the device performance.

Sohrabpour et al. demonstrated that SFG spectra collected from $\mathrm{C}_{60}$ can be interfered by surface charge on dielectric layers. ${ }^{[26]}$ For the same materials used, different dielectric substrates used in semiconducting devices may cause the SFG spectrum of $\mathrm{C}_{60}$ to differ. $\mathrm{C}_{60}$ is also widely used in halide perovskite photovoltaic applications as an ETM. From this work, SFG demonstrated its ability to study $\mathrm{C}_{60}$ S used as ETLs in halide perovskite devices.

Dhar et al. discovered that annealing polymeric semiconducting materials could induce changes of the buried interfacial structure. ${ }^{[2]}$ Thermal annealing is one of the most commonly used methods to enhance surface contacts and influences the
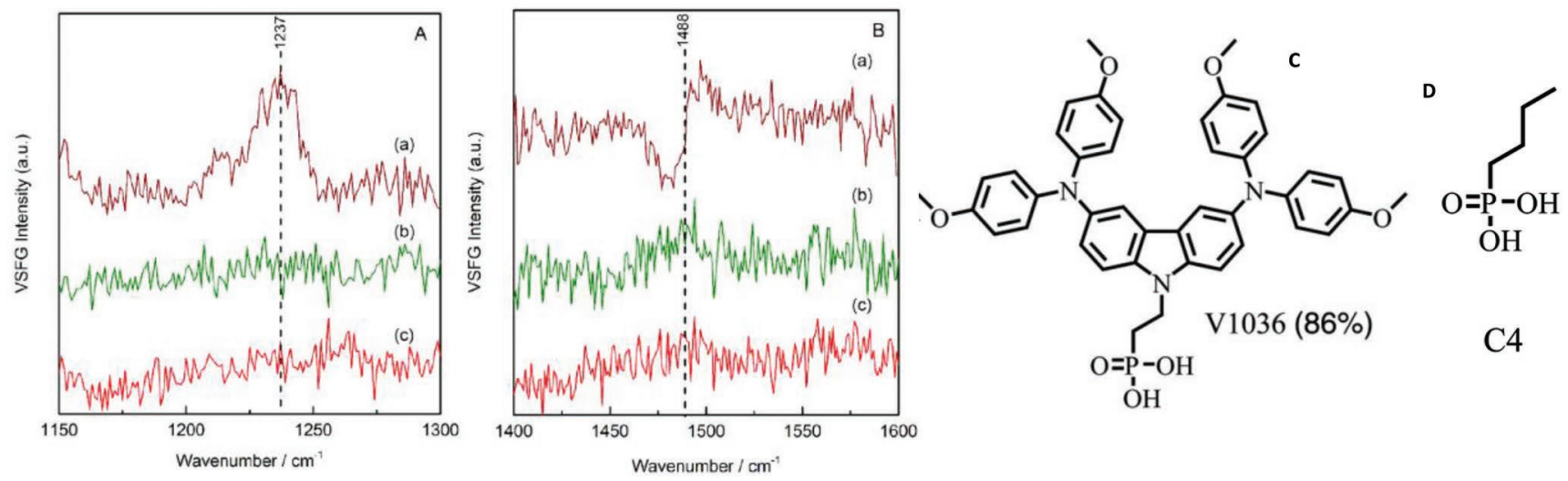

C4

Figure 8. A,B) SFG spectra in different frequency ranges collected from monolayers on Si/ITO substrate prepared from $(\mathrm{A}) 1 \times 10^{-3} \mathrm{M}$ solution of V1036, (B) $1 \times 10^{-3} \mathrm{M}$ of mixed solution V1036:C4 (1:9), and (C) $1 \times 10^{-3} \mathrm{M}$ solution of C4. C,D) Molecular formulas for V1036 and C4. Adapted with permission. ${ }^{[24]}$ Copyright 2018, Wiley. 
overall device performance in perovskite photovoltaic applications. Dhar et al.'s results suggest that the enhancement of "interfacial contact" may actually lead to the interfacial orientation change of polymeric materials. ${ }^{[2]}$ In perovskite solar cells, such buried interfacial orientation changes could result in variation of the overall device PCE.

As discussed in Section 3.2 above, buried interfacial SAM structure can be used to help optimize the overall device performance. The work by Anglin et al. suggests that different SAMs may simultaneously influence the interfacial structures of the materials in contact with the SAM surface. ${ }^{[28]}$ This will further lead to different device performances. Anglin et al. reported that the same semiconducting material may adopt diverse interfacial orientations at various buried dielectric material interfaces due to different interfacial interactions. ${ }^{[28]}$ This will lead to drastic differences in overall hole transporting abilities.

The above SFG results clearly demonstrate that solvent composition, sample preparation methods such as annealing, and substrate dielectric materials can be systematically varied to gain general knowledge on molecular buried interfacial structures. Such knowledge is important for the design and optimization of interfacial structures in perovskite devices to improve device performance. We believe that more and more important SFG research on buried interfaces in perovskite related materials and devices will be performed in the future.

\section{Summary and Outlook}

It is important to examine the buried interfacial structures of halide perovskite solar cells in situ and at the molecular level with monolayer sensitivity. It is difficult for the current widely used analytical techniques in perovskite research to do so. SFG probes surfaces and interfaces with sub-monolayer sensitivity, and provides vibrational spectra of buried solid/solid interfaces in situ nondestructively. From these spectra, molecular structure information (e.g., interfacial presence and orientation of various functional groups) can be obtained.

SFG has been applied to study buried interfaces between perovskite and PT HTLs. It was found that the PT orientation at the buried perovskite/HTL interface can be varied through PT side chains of different lengths. A standing-up interfacial PT backbone leads to a high conversion efficiency of perovskite solar cells. This result provided important knowledge to design HTL materials for a better quality perovskite solar cell, with a high conversion efficiency of $19.68 \%$. SFG studies also indicated that disordered orientation of small SAM molecules at the perovskite/HTM interface leads to high conversion efficiency in perovskite solar cells. These SFG studies further demonstrate the significance of using SFG to study interfaces within halide perovskite devices, which can provide vital knowledge to guide material design for building halide perovskite devices with improved performance. More SFG studies on perovskite materials at buried ETM and HTM interfaces should be performed to understand how various ETM and HTM layers impact interfacial perovskite materials and further influence perovskite solar cell PCEs. To avoid confusion of signal contributions from ETM and HTM layers, deuterated ETL/HTL materials/polymers may need to be used.
It is necessary to mention that interfacial orientation or ordering is not the only factor which dictates the perovskite photovoltaic performance. Many other factors besides interfacial orientation (e.g., interfacial defects and ionization potentials) can profoundly influence the perovskite photovoltaic performance. Such factors may or may not be connected to the interfacial orientation or ordering. More detailed and systematic studies in the future on various factors at the interface will give us a more completed picture to understand the interfacial structure-function relationships of perovskite photovoltaics.

It is also worth mentioning that in order to apply SFG to elucidate molecular structure of a buried interface, the two input laser beams need to reach the buried interface. This may not be always possible, especially for thick opaque samples. We have developed a "milling down" approach to polish the sample to about $1 \mu \mathrm{m}$ above the interface in a thick sample. ${ }^{[29]}$ The input beams can then penetrate through the top 1 micrometer layer to probe the buried interface. This method can also be applied to study buried interfaces of thick multilayered films to study different interfaces between various layers. ${ }^{[29]}$

We wish that this article introduces and motivates the halide perovskite research community to use the powerful SFG technique. We also hope that it will open up room for more discussion and collaboration between SFG analytical scientists, halide perovskite material/device scientists, and organic synthetic chemists. By fully understanding different interfaces of a halide perovskite device, we then can rationally design and develop interfaces in halide perovskite photovoltaic devices to improve the overall device performance.

\section{Acknowledgements}

This research was supported by the University of Michigan.

\section{Conflict of Interest}

The authors declare no conflict of interest.

\section{Keywords}

buried interfaces, molecular structures, perovskite photovoltaics, semiconducting polymers, sum frequency generation vibrational spectroscopy

Received: September 17, 2019

Revised: November 5, 2019

Published online: November 26, 2019

[1] a) A. K. Jena, A. Kulkarni, T. Miyasaka, Chem. Rev. 2019, 119, 3036; b) L. M. Quan, B. P. Rand, R. H. Friend, S. G. Mhaisalkar, T.-W. Lee, E. H. Sargent, Chem. Rev. 2019, 119, 7444.

[2] a) M. Liu, M. B. Johnston, H. J. Snaith, Nature 2013, 501, 395; b) S. T. Ha, X. Liu, Q. Zhang, D. Giovanni, T. C. Sum, Q. Xiong, Adv. Opt. Mater. 2014, 2, 838; c) E. Edri, S. Kirmayer, S. Mukhopadhyay, K. Gartsman, G. Hodes, D. Cahen, Nat. Commun. 2014, 5, 3461; d) Q. Chen, H. Zhou, Z. Hong, S. Luo, H.-S. Duan, H.-H. Wang, 
Y. Liu, G. Li, Y. Yang, J. Am. Chem. Soc. 2014, 136, 622; e) A. Oranskaia, U. Schwingenschlogl, Adv. Energy Mater. 2019, 9, 1901411; f) Y. Liu, Z. Yang, S. F. Liu, Adv. Sci. 2018, 5, 1700471.

[3] a) J. Li, T. Jiu, C. Duan, Y. Wang, H. Zhang, H. Jian, Y. Zhao, N. Wang, C. Huang, Y. Li, Nano Energy 2018, 46, 331; b) A. Krishna, D. Sabba, H. Li, J. Yin, P. P. Boix, C. Soci, S. G. Mhaisalkar, A. C. Grimsdale, Chem. Sci. 2014, 5, 2702; c) Q. Wang, E. Mosconi, C. Wolff, J. Li, D. Neher, F. De Angelis, G. P. Suranna, R. Grisorio, A. Abate, Adv. Energy Mater. 2019, 9, 1900990; d) Y. Feng, Q. Hu, E. Rezaee, M. Li, Z.-X. Xu, A. Lorenzoni, F. Mercuri, M. Muccini, Adv. Energy Mater. 2019, 9, 1901019.

[4] a) D. Forgács, L. Gil-Escrig, D. Pérez-Del-Rey, C. Momblona, J. Werner, B. Niesen, C. Ballif, M. Sessolo, H. J. Bolink, Adv. Energy Mater. 2017, 7, 1602121; b) S. Gharibzadeh, B. A. Nejand, M. Jakoby, T. Abzieher, D. Hauschild, S. Moghadamzadeh, J. A. Schwenzer, P. Brenner, R. Schmager, A. A. Haghighirad, L. Weinhardt, U. Lemmer, B. S. Richards, I. A. Howard, U. W. Paetzold, Adv. Energy Mater. 2019, 9, 1803699.

[5] P. Schulz, D. Cahen, A. Kahn, Chem. Rev. 2019, 119, 3349.

[6] M. Xiao, S. Joglekar, X. Zhang, J. Jasensky, J. Ma, Q. Cui, L. J. Guo, Z. Chen, J. Am. Chem. Soc. 2017, 139, 3378.

[7] J. A. Christians, P. Schulz, J. S. Tinkham, T. H. Schloemer, S. P. Harvey, B. J. T. de Villers, A. Sellinger, J. J. Berry, J. M. Luther, Nat. Energy 2018, 3, 68.

[8] Y. Yang, Q. Chen, Y.-T. Hsieh, T.-B. Song, N. D. Marco, H. Zhou, ACS Nano 2015, 9, 7714.

[9] S. Yang, J. Dai, Z. Yu, Y. Shao, Y. Zhou, X. Xiao, X. C. Zeng, J. Huang, J. Am. Chem. Soc. 2019, 141, 5781.

[10] Y. Hou, X. Du, S. Scheiner, D. P. McMeekin, Z. Wang, N. Li, M. S. Killian, H. Chen, M. Richter, I. Levchuk, N. Schrenker N, E. Spiecker, T. Stubhan, N. A. Luechinger, A. Hirsch, P. Schmuki, H. P. Steinrück, R. H. Fink, M. Halik, H. J. Snaith, C. J. Brabec, Science 2017, 358, 1192.

[11] J. J. Yoo, S. Wieghold, M. C. Sponseller, M. R. Chua, S. N. Bertram, N. T. P. Hartono, J. S. Tresback, E. C. Hansen, J.-P. Correa-Baena, V. Bulović, T. Buonassisi, S. S. Shin, M. G. Bawendi, Energy Environ. Sci. 2019, 12, 2192.

[12] C. M. Wolff, P. Caprioglio, M. Stolterfoht, D. Neher, Adv. Mater. 2019, 1902762.

[13] a) G. Grancini, V. D'Innocenzo, E. R. Dohner, N. Martino, A. S. Kandada, E. Mosconi, F. De Angelis, H. Karunadasa, E. Hoke, A. Petrozza, Chem. Sci. 2015, 6, 7305; b) Q. Dong, F. Liu, M. K. Wong, H. W. Tam, A. B. Djurišić, A. Ng, C. Surya, W. K. Chan, A. M. C. Ng, ChemSusChem 2016, 9, 2597.

[14] Y. Shen, Nature 1989, 337, 519.

[15] a) Y. R. Shen, Annu. Rev. Phys. Chem. 1989, 40, 327; b) K. B. Eisenthal, Chem. Rev. 1996, 96, 1343; c) M. Buck, M. Himmelhaus, J. Vac. Sci. Technol., A 2001, 19, 2717; d) Z. Chen, Y. R. Shen, G. A. Somorjai, Annu. Rev. Phys. Chem. 2002, 53,
437; e) C. T. Williams, D. A. Beattie, Surf. Sci. 2002, 500, 545; f) G. L. Richmond, Chem. Rev. 2002, 102, 2693; g) F. M. Geiger, Annu. Rev. Phys. Chem. 2009, 60, 61; h) Z. Chen, Prog. Polym. Sci. 2010, 35, 1376; i) E. C. Y. Yan, L. Fu, Z. Wang, W. Liu, Chem. Rev. 2014, 114, 8471; j) S. Roy, K.-K. Hung, U. Stege, D. K. Hore, Appl. Spectrosc. Rev. 2014, 49, 233; k) B. Ding, J. Jasensky, Y. Li, Z. Chen, Acc. Chem. Res. 2016, 49, 1149; I) D. Hu, K. Chou, J. Am. Chem. Soc. 2014, 136, 15114; m) H. Wang, W. Chen, J. C. Wagner, W. Xiong, J. Phys. Chem. B 2019, 123, 6212; n) S.-Y. Jung, S.-M. Lim, F. Albertorio, G. Kim, M. C. Gurau, R. D. Yang, M. A. Holden, P. S. Cremer, J. Am. Chem. Soc. 2003, 125, 12782.

[16] X. Zhuang, P. Miranda, D. Kim, Y. Shen, Phys. Rev. B 1999, 59, 12632.

[17] X. Lu, C. Zhang, N. Ulrich, M. Xiao, Y.-H. Ma, Z. Chen, Anal. Chem. 2017, 89, 466.

[18] a) W. R. FitzGerald, K. C. Jena, D. K. Hore, J. Mol. Struct. 2015 1084, 368; b) P. M. Kearns, D. B. O'Brien, A. M. Massari, J. Phys. Chem. Lett. 2016, 7, 62; c) X. Lu, D. Li, C. B. Kristalyn, J. Han, N. Shephard, S. Rhodes, G. Xue, Z. Chen, Macromolecules 2009, 42, 9052; d) X. Lu, G. Xue, X. Wang, J. Han, X. Han, J. Hankett, D. Li, Z. Chen, Macromolecules 2012, 45, 6087; e) J. N. Myers, X. Zhang, J. Bielefeld, Q. Lin, Z. Chen, J. Phys. Chem. B 2015, 119, 1736; f) X. Zhang, J. N. Myers, H. Huang, H. Shobha, Z. Chen, A. Grill, J. Appl. Phys. 2016, 119, 084101; g) X. Lu, N. Shephard, J. Han, G. Xue, Z. Chen, Macromolecules 2008, 41, 8770

[19] L. Zhang, C. Liu, J. Zhang, X. Li, C. Cheng, Y. Tian, A. K. Y. Jen, B. Xu, Adv. Mater. 2018, 30, 1804028.

[20] C. Hirose, N. Akamatsu, K. Domen, Appl. Spectrosc. 1992, 46, 1051.

[21] a) C. Hirose, H. Yamamoto, N. Akamatsu, K. DomenJ. Phys. Chem. 1993, 97, 10064; b) C. Hirose, N. Akamatsu, K. DomenJ. Chem. Phys. 1992, 96, 997.

[22] A. J. Moad, G. J. Simpson, J. Phys. Chem. B 2004, 108, 3548.

[23] E. H. Jung, N. J. Jeon, E. Y. Park, C. S. Moon, T. J. Shin, T.-Y. Yang, J. H. Noh, J. Seo, Nature 2019, 567, 511.

[24] A. Magomedov, A. Al-Ashouri, E. Kasparavičius, S. Strazdaite, G. Niaura, M. Jošt, T. Malinauskas, S. Albrecht, V. Getautis, Adv. Energy Mater. 2018, 8, 1801892.

[25] M. Xiao, X. Zhang, Z. J. Bryan, J. Jasensky, A. J. McNeil, Z. Chen, Langmuir 2015, 31, 5050.

[26] Z. Sohrabpour, P. M. Kearns, A. M. Massari, J. Phys. Chem. C 2016, 120, 1666.

[27] P. Dhar, P. P. Khlyabich, B. Burkhart, S. T. Roberts, S. Malyk, B. C. Thompson, A. V. Benderskii, J. Phys. Chem. C 2013, 117, 15213.

[28] T. C. Anglin, Z. Sohrabpour, A. M. Massari, J. Phys. Chem. C 2011, 115,20258

[29] N. W. Ulrich, M. Xiao, X. Zou, J. Williamson, Z. Chen, IEEE Trans. Compon., Packag., Manuf. Technol. 2018, 8, 1213. 